\title{
Foreword: An Exploration-Driven Renaissance in Lunar Science
}

\author{
Wendell Mendell
}

Received: 17 February 2010 / Accepted: 17 February 2010 / Published online: 2 March 2010

(C) Springer Science+Business Media B.V. 2010

In the $3 \frac{1}{2}$ years between the first human lunar landing and the final launch, the Apollo engineering team enormously expanded the range and performance of surface systems and added orbital mapping capabilities. High-resolution cameras and a laser altimeter in the Service Module Bay photographed and profiled the lunar surface, including areas that might be future Apollo landing sites. A gamma ray spectrometer and an X-ray spectrometer provided data for construction of maps indicating the concentration of certain elements. In today's mode of doing business, a similar systems evolution would take a decade or more.

While the gains in surface mobility, astronaut EVA time, and precision landing were impressive and productive, the first geochemical (elemental) maps from the orbital spectrometers startled many planetary scientists. Those maps could be interpreted in terms of the rock types identified in the returned samples. In principle, scientists could begin to infer the geological evolution of a planet had the mapping been global.

To that end, leaders in the lunar science community approached NASA about the addition of a small, unmanned polar orbiter mission. When the Administrator suggested that Apollo 17 could be an orbit-only mission, the conversation ceased.

In 1973, the Manned Spacecraft Center (now the Johnson Space Center) submitted an unsolicited proposal to NASA Headquarters for an inexpensive lunar polar orbiter with only geochemical instruments, i.e., no cameras. The Office of Space Science was taken by surprise and eventually decided to compete the mission. Of the three NASA Centers that proposed, MSC had the most robust lunar science capability; but the evaluation board doubted that a human spaceflight center would be capable of cost containment on a small spacecraft project. The mission was first assigned to the Goddard Space Flight Center and, after a year of little progress, was transferred to the Jet Propulsion Laboratory. There, the mission acquired the designation Terrestrial Bodies Orbiter-Lunar (TBOL) to be consistent with the recently issued report of the Terrestrial Bodies Science Working Group. That advisory com-

W. Mendell ( $\varangle)$

NASA/JSC, Houston, TX, USA

e-mail: wendell.w.mendell@nasa.gov 
mittee had drawn up a comprehensive exploration plan for all "rocky" bodies in the solar system.

The JPL Director had made a famous speech to his employees, urging them to conceive of visually exciting, Congressionally-attractive missions to far flung destinations using the latest technology with lots of imaging (which he called "purple pigeons"), in contrast to mundane science spacecraft sent to nearby destinations (which he called "grey mice"). A T-shirt was sold that year at the Lunar Science Conference depicting a purple pigeon and a grey mouse attempting to coexist on a cratered terrain. JPL nevertheless produced a fine lunar spacecraft design with a capable science team before the project somehow faded away. The reports are still in my file cabinet.

Thus began a 30-year drought on lunar initiatives from the Office of Space Science. However, the lunar science community had not given up on a new lunar mission.

European scientists unsuccessfully proposed an ESA Polar Orbiting Lunar Observatory (POLO). Italians reworked the concept into a Moon Orbiting Observatory (MORO) proposal to their space agency invoking a launch from the Space Shuttle. A Lunar Geoscience Observer appeared in a list of second tier recommendations for a new line of planetary spacecraft in the 1983 report to NASA from the Solar System Exploration Committee. The failure and the high cost of the Mars Observer doomed the new program and any possible lunar mission.

At the Lunar Science Conference in 1987, Dr. Tom McCord chaired an informal meeting of international lunar scientists to formulate a coordinated strategy for proposing lunar missions to three major space agencies. The discussion went so far as to "allocate" different themes to the various national groups represented in the room. Each contingent planned to approach their own agency with a lunar mission concept and point out a complementary concept being considered in another agency. Professor Hitoshi Mizutani was one of the Japanese representatives, and his successful Lunar-A proposal to ISAS was the only tangible outcome of the conspiracy. Unfortunately, his mission development, built around penetrators with geophysical instrumentation, faced formidable technical challenges and was never flown.

Two years later, a U.S. President stood on the steps of the National Air and Space Museum and proposed a broad program of human exploration, within which the Moon would be an important element. During the brief existence of the Space Exploration Initiative, Associate Administrator for Exploration Michael Griffin championed an inexpensive, fast-track project called Lunar Scout for orbital surveys. Its track was not fast enough, and it vanished in the political storm that sank the SEI ship.

In 1994, a U.S. spacecraft arrived in lunar orbit carrying an array of sensors developed within the Department of Defense, with some guidance from NASA and the planetary science community. The mission, described as an in-space technology demonstration, was the brainchild of Pete Worden, then an Air Force colonel and later Director of the Ames Research Center. Of particular importance was the multispectral imaging system and the lidar rangefinder device that could precisely determine the distance between the spacecraft and the physical surface beneath it with $20 \mathrm{~m}$ to $30 \mathrm{~m}$ resolution. For 71 days, Clementine mapped the Moon. The data from the laser ranging device significantly improved our knowledge of lunar topography, and from the multispectral imagery were constructed the first global maps of lunar mineralogy. Many people, particularly from outside the U.S., erroneously believe that Clementine was a NASA mission.

The Clementine operations team noted that two specific contiguous orbits passing over the lunar south pole were oriented with respect to the Earth so as to allow a bistatic "radar" measurement potentially capable of detecting pure water ice inside permanently shadowed 
craters. During those specific passes, an Earth-based antenna monitored the spacecraft's telemetry signal as it was reflected from the lunar surface. Similar bistatic measurements had been performed during the Apollo 15 and 16 missions, though not over the polar regions. The team published results claiming to observe characteristics of the expected water ice signal in the coherent backscatter opposition effect associated with the reflected beam. A subsequent paper by different authors failed to find the reported signature in a reexamination of the data. The lively controversy over the bistatic radar result lives on today.

In 1994 NASA instituted a new program, named Discovery, to create a line of low-cost planetary mission spacecraft with associated scientific investigations. Lunar Prospector was the third Discovery mission but was the first to be competitively selected from team proposals led by a Principal Investigator. Its selection was largely attributed to an extraordinarily low cost estimate in an era whose motto was "faster, better, cheaper". Some senior members of the lunar science community publicly complained that such a spartan spacecraft could not possibly yield data of any utility and might "kill" lunar science for another 30 years. However, the unusual spin-stabilized polar orbiter performed admirably and produced major discoveries at its promised low cost. The Lunar Prospector gamma ray spectrometer mapped out a large region rich in radioactive elements, undoubtedly the source of the enigmatic KREEP component found in the Apollo samples. (KREEP is a quasi-acronym for an enigmatic geochemical component of Apollo samples that was rich in potassium, rare earth elements, and phosphorus.) The discovery of the Procellarum KREEP Terrane (PKT) solved a geochemical mystery in the lunar samples but toppled simplistic spherically symmetric scenarios of lunar thermal evolution. Meanwhile, the Lunar Prospector Neutron Spectrometer was returning valuable information on unanticipated concentrations of hydrogen in the lunar surface layer at the poles. Although the spatial resolution of the data is very coarse, oversampling in the polar regions permits investigators to suggest tantalizing relationships of the proton excess with the interiors of large, permanently shadowed craters, adding fuel to the "lunar ice" fire.

In January 2004, another President of the United States reincarnated human exploration of the solar system with a surprisingly detailed charge to NASA that featured lunar exploration as a major near-term theme. On March 1, 2004, senior members of the lunar science community convened a workshop at the Lunar and Planetary Institute (LPI) to discuss what characteristics of the lunar environment should be determined by the first precursor lunar orbital mission for the new initiative. Workshop chair G. Jeffrey Taylor reminded the attendees that policy goals gave priorities to support of human exploration over those of pure science, even though lunar science would clearly benefit. On the other hand, NASA Chief Scientist Jim Garvin (by phone) urged the attendees not to neglect advancement of lunar science in their recommendations because of its increasing relevance to the understanding of the entire Solar System.

Working from these somewhat discordant charges, all attendees agreed that the first priority was to ensure a safe landing and return of human explorers. Much better photography as well as topographic and cartographic mapping was needed for the Moon to pick landing sites and to enable precise navigation. (Photographic coverage of Mars was much better than the lunar data set, much to the astonishment of NASA engineers.) Next on the list was the ability to find and characterize resources as an aid to extended human presence and for future lunar economic development. From the perspective of exploration, any polar volatiles and the nature of the polar environment must be understood. Direct measurement of surface temperature was desirable, particularly in the unseen depths of the permanently shadowed polar craters. The radiation environment must be monitored to model its effects on longduration human lunar missions. As the lunar surface directly experiences the solar wind plasma, surface electrical fields also were of some concern. 


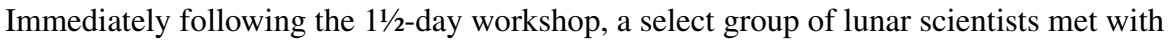
Chief Scientist Garvin to begin the process of defining the robotic mission that would be called Lunar Reconnaissance Orbiter. Of course, moving from a set of data needs to a mission concept with an instrument complement involves a number of considerations such as instrument technology readiness, budget, and schedule. The LRO mission as flown represents certain compromises, but every instrument onboard addresses the needs of future exploration in some way, and most are clearly tied to the priorities articulated by the March meeting attendees. Indeed, many of the attendees of the March 1, 2004 workshop would never have believed that LRO could obtain sub-meter resolution imaging, $10 \mathrm{~km}$ scale hydrogen mapping, 5-10 cm vertical accuracy topographic mapping, and temperature mapping down to $20 \mathrm{~K}$ !

As the LRO project gathered steam, the lunar community witnessed a multinational fleet of spacecraft arriving at the Moon. The first to arrive was Small Missions for Advanced Research in Technology One (SMART-1) in November 2004 from ESA. Using ion engines for a slow but highly fuel-efficient lunar transfer orbit, the small spacecraft carried a suite of instruments intended as proof of technologies. Next to attain orbit was the huge Kaguya spacecraft from JAXA, which entered its operational orbit in December 2007. Its impressive suite of instruments mapped the Moon for 18 months. With its two sibling satellites Okina and Ouna, it performed the only true mapping of the lunar farside gravity field. The Kaguya gravity field model and the topography data has been used to plan the LCROSS impactor mission that was selected to ride along with LRO. Chang'e from China arrived shortly after Kaguya and quickly began orbital operations from a 200-km altitude, mapping for 16 months. Approximately a year later, India's Chandrayaan arrived and began collecting information at its planned 100-km altitude. After LRO arrived on the scene in June 2009, it coordinated observations with the Indian spacecraft to perform a two-spacecraft version of the Clementine bistatic measurement of the reflected signal from the interior of polar craters. Unfortunately the experiment was unsuccessful.

The missions of Smart-1, Kaguya, and Chang'e ended with controlled impacts into the lunar surface, while Chandrayaan collected data up until a communications failure. Japan, China, and India have announced plans for follow-on lunar missions with landings on the surface.

As of this writing, the Augustine Commission Report is in preparation and will be an important element in a review of current priorities in U.S. civilian space policy, including reassessment of human exploration of the Moon. Irrespective of that decision, we can now say that the polar orbiter mission conceived at the end of the Apollo Program has finally been flown, not once but several times! We have new participants in lunar exploration, both international space agencies and private entities, whose motivations and interests are not directly tied to NASA programs. Data from LRO will be placed in the public domain expeditiously, and we hope that the findings (and particularly the measurements) from other missions will also be accessible to the international scientific community. Privately financed operations are under no such imprimatur for disclosure of results, but such missions can carry scientific instruments as customers. I am optimistic that this panoply of new activity will generate a great deal of new understanding of the history and current state of the Moon and will engender an exploration-driven renaissance of lunar science. 